Article

\title{
Investigation of the Impact of Cold Plasma Treatment on the Chemical Composition and Wettability of Medical Grade Polyvinylchloride
}

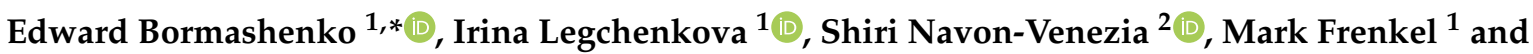 \\ Yelena Bormashenko ${ }^{1}$ \\ 1 Chemical Engineering Department, Engineering Sciences Faculty, Ariel University, Ariel 407000, Israel; \\ ilegchenkova@gmail.com (I.L.); markfr@ariel.ac.il (M.F.); Yelenabo@ariel.ac.il (Y.B.) \\ 2 Department of Molecular Biology, Faculty of Natural Sciences and the Adelson School of Medicine, \\ Ariel University, Ariel 40700, Israel; shirinv@ariel.ac.il \\ * Correspondence: edward@ariel.ac.il; Fax: +972-3-9366621
}

check for updates

Citation: Bormashenko, E.; Legchenkova, I.; Navon-Venezia, S.; Frenkel, M.; Bormashenko, Y. Investigation of the Impact of Cold Plasma Treatment on the Chemical Composition and Wettability of Medical Grade Polyvinylchloride. Appl. Sci. 2021, 11, 300. https:// doi.org/10.3390/app11010300

Received: 22 November 2020 Accepted: 25 December 2020 Published: 30 December 2020

Publisher's Note: MDPI stays neutral with regard to jurisdictional clai$\mathrm{ms}$ in published maps and institutional affiliations.

Copyright: (C) 2020 by the authors. Licensee MDPI, Basel, Switzerland. This article is an open access article distributed under the terms and conditions of the Creative Commons Attribution (CC BY) license (https:// creativecommons.org/licenses/by/ $4.0 /)$.

\begin{abstract}
The impact of the Corona, dielectric barrier discharge, and low pressure radiofrequency air plasmas on the chemical composition and wettability of medical grade polyvinylchloride was investigated. Corona plasma treatment exerted the most pronounced increase in the hydrophilization of polyvinylchloride. The specific energy of adhesion of the pristine and plasma-treated Polyvinylchloride (PVC) tubing is reported. Plasma treatment increased markedly the specific free surface energy of PVC. The kinetics of hydrophobic recovery following plasma treatment was explored. The time evolution of the apparent contact angle under the hydrophobic recovery is satisfactorily described by the exponential fitting. Energy-dispersive X-ray spectroscopy of the chemical composition of the near-surface layers of the plasma-treated catheters revealed their oxidation. The effect of the hydrophobic recovery hardly correlated with oxidation of the polymer surface, which is irreversible and it is reasonably attributed to the bulk mobility of polymer chains.
\end{abstract}

Keywords: cold plasma; polyvinylchloride; wettability; hydrophilization; apparent contact angle; contact angle hysteresis; hydrophobic recovery

\section{Introduction}

A diversity of engineering applications calls for the modification of the surface properties of industrial polymer materials, including their adhesion and wettability. These applications include packaging, protective coatings, adhesives, textile, printing, etc. In the fields of medicine and biotechnology, many products such as catheters and medical implants are completely or partly made of polymers. In contact with biological systems, compatibility of these materials is not always specified. Medical applications often demand the modification of the properties of polymer surfaces. Plasma techniques supply an appropriate tool for the generation of demanded surface properties [1-6]. Plasma modified materials fulfil the requirements for bioactivity in medicine; for example, the inclusion of antimicrobial agents (metal nano-particles, antimicrobial peptides, enzymes, etc.) in plasma- modified materials (polymeric, metallic, etc.) alters them to produce superior antibacterial biomedical devices with a longer active life [4].

Various kinds of discharges were already applied for the surface modification of medical polymers including the atmospheric [7] and low pressure plasma discharges [8]. The plasma treatment creates a complex mixture of surface groups, constituting surface physical and chemical properties, which results in an essential increase in the specific surface free energy and consequent change in the wetting properties of the plasma modified surface [9-12]. It was demonstrated recently that the plasma treatment may result in the cross-linking of polymer molecules [13]. It was reported that plasma treatment leads to the 
essential electrical charging of a polymer surface observed for both synthetic and biological polymers $[14,15]$. It is agreed that the cold plasma modification increases the specific surface energy of polymers, thus resulting in their pronounced hydrophilization [16-21]. At the same time the precise physico-chemical mechanism of this hydrophilization remains debatable. It was suggested that the hydrophilization of organic surfaces by plasmas may be at least partially related to the re-orientation of hydrophilic moieties constituting organic molecules [22,23]. The oxidation of plasma-treated surfaces and removal of low-mass fragments present on organic surfaces also contribute to hydrophilization [24,25].

It was reported that the specific surface energy of cold plasma-treated polymers decreases with time and consequently hydrophilization provided by plasma treatment is partially lost with time [26-30]. This process is called hydrophobic recovery [26-30]. It was demonstrated experimentally that hydrophobic recovery is stipulated by the reorientation of the polar groups constituting the polymer chains, which were oriented by the plasma treatment [28]. A model for the hydrophobic recovery due to a combination of two thermodynamically non-equilibrium processes, diffusion and molecular reorientation, was suggested [30]. Our manuscript focuses on the estimation of influence of cold plasma treatment on the wettability and hydrophobic recovery of medical grade Polyvinylchloride (PVC), broadly used for manufacturing of medical catheters. Cold plasma treatment of PVC was reported by several groups [31-34], however the experimental data related to this polymer remain sparse. We report a comparative study of the impact of various plasma discharges, including the Corona discharge, dielectric barrier (DBD) discharge, and radiofrequency low pressure air plasma discharge on the wettability and hydrophobic recovery of medical grade PVC.

\section{Materials and Methods}

\subsection{Materials}

PVC CH18 suction catheters (diameter $6 \mathrm{~mm}$, length $53 \mathrm{~cm}$ ) supplied by Unomedical ConvaTec Lim. (Deeside, UK) were plasma treated in the experiments. For the purposes of the goniometric measurements of wettability, the catheters were flattened under $t=80^{\circ} \mathrm{C}$ and pressure of $1 \mathrm{kPa}$ during $40 \mathrm{~min}$, followed by cooling to ambient conditions $\left(t=25 \pm 1{ }^{\circ} \mathrm{C}\right)$ over $1 \mathrm{~h}$.

The de-ionized water, used for the study of the wettability of catheters, was purified by a synergy UV water purification system from Millipore SAS (Molsheim, France) and its specific resistivity was $\hat{\rho}=18.2 \mathrm{M} \Omega \times \mathrm{cm}$ at $25^{\circ} \mathrm{C}$.

\subsection{Methods}

The Corona plasma device (3DT, MULTIDYNE 1000, Germantown, WI, USA) consisted of a treating head that contained two hook-shaped wire electrodes. The plasma was generated under high voltage at an electrode of $2 \times 12 \mathrm{kV}$ and a frequency of $50 \mathrm{~Hz}$ at atmospheric pressure conditions, using ambient air as a carrier gas. The distance between the electrode and flattened catheter was $2 \pm 0.1 \mathrm{~cm}$.

The parameters of an air low pressure radiofrequency plasma discharge were: The plasma frequency was $13.56 \mathrm{MHz}$; the power was $18 \mathrm{~W}$; the pressure was 2 Torr; the times of irradiation varied at 10,20 , and $30 \mathrm{~s}$; and the volume of the discharge chamber was $840 \mathrm{~cm}^{3}$. Dried compressed air used for the radiofrequency plasma treatment of catheters was supplied by Oxygen \& Argon Works, Ltd. (Caesarea, Israel), moisture was less than $10 \mathrm{ppm}$, and the concentration of oxygen was $20-22 \%$.

The diffuse coplanar surface barrier discharge was created by the DBD plasma unit RPS 40, supplied by Roplass (Brno, Czech Pepublic), the maximal power was $40 \mathrm{~W}$, and frequency $\sim 20 \mathrm{KHz}$. The distance between the electrode and flattened catheter was $0.5 \mathrm{~mm}$. The time span of the plasma treatment varied within 15-60 s.

Apparent contact angles were established using the Ramé-Hart goniometer (Model 500). A typical picture of a water droplet placed on a pristine PVC catheter is shown in Figure 1. A total of 8 measurements were taken to calculate mean apparent contact angles 
at ambient conditions. The apparent contact angles were taken on both sides of a droplet and the results were averaged.

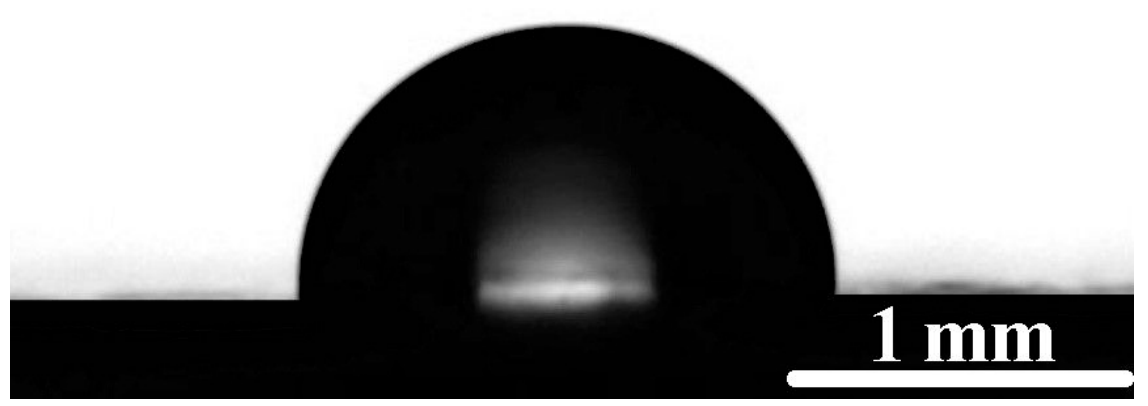

Figure 1. Wettability of pristine Polyvinylchloride (PVC) catheter is illustrated. The apparent contact angle is close to $\frac{\pi}{2}$.

Contact angle hysteresis was established with the tilted plane method. A $5 \mu \mathrm{L}$ water droplet was placed on the PVC sample mounted on the glass slide. The slide was tilted until the drop began to move. The front and rear contact angles at which the droplet started to slip are regarded as the advancing $\theta_{a d v}$ and receding $\theta_{\text {rec }}$ contact angles, correspondingly, as depicted in Figure 2.

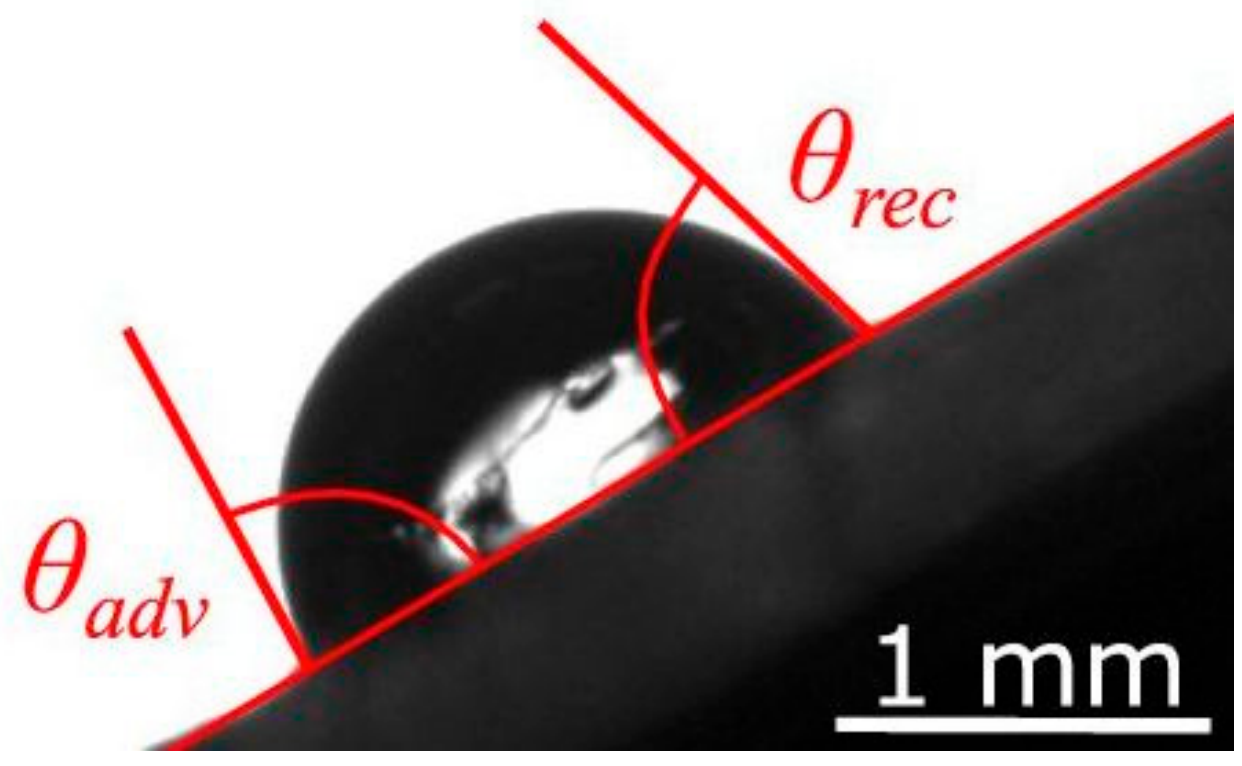

Figure 2. Water droplet placed on the tilted PVC surface is shown. The advancing $\theta_{a d v}$ and receding $\theta_{\text {rec }}$ contacting angles are depicted.

The difference $\Delta \theta=\theta_{a d v}-\theta_{\text {rec }}$ is called the contact angle hysteresis [35-39]. In spite of the fact that the "tilted plane experiment" was criticized as a method for the accurate establishment of the contact angle hysteresis, it is still broadly used for its estimation [39,40]. In parallel, the contact angle hysteresis was measured with a needle-syringe method.

The $5 \mu \mathrm{L}$ water droplet placed on the flattened catheter was inflated with a syringe as shown in Figure 3A. When the contact (triple line) was pinned and the contact angle was increased till a certain threshold value beyond which the triple line moved. This threshold contact angle $\theta_{\text {adv }}$ was regarded as the advancing contact angle [35-39]. When a droplet was deflated as depicted in Figure 3B, its volume decreased to a certain limiting value; in parallel the contact angle decreases till a threshold value $\theta_{\text {rec }}$, interpreted as the receding contact angle [35-39]. 


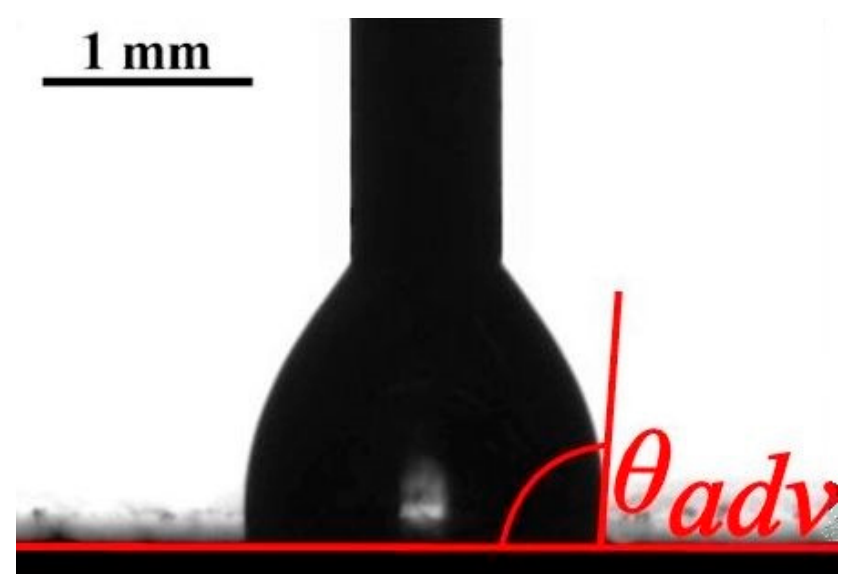

(A)

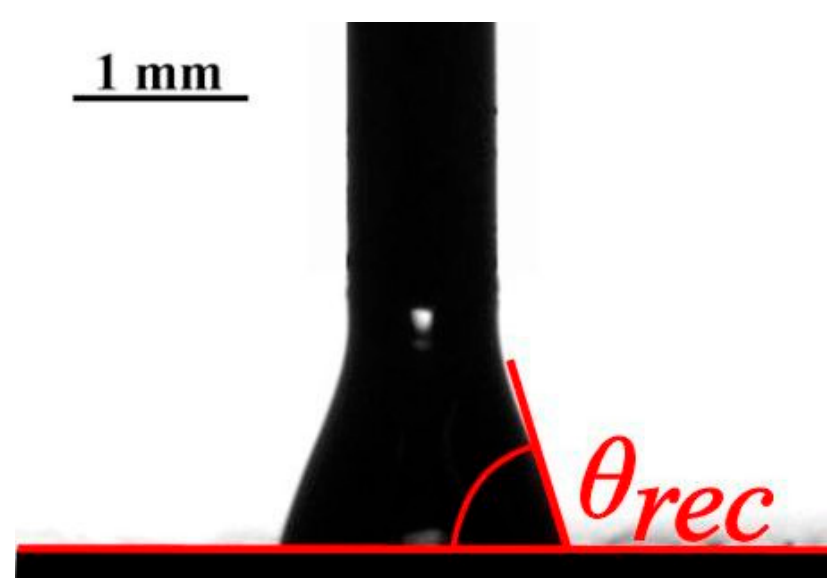

(B)

Figure 3. Establishment of the contact angle hysteresis on the pristine PVC catheter is demonstrated. (A) Measurement of the advancing contact angle is shown. (B) Measurement of the receding contact angle is depicted.

The chemical composition of the pristine and cold plasma-treated PVC catheters was studied with SEM/EDS (scanning electron microscopy/energy dispersive spectrometry) carried out with SEM (MAIA3 TESCAN).

\section{Results and Discussion}

\subsection{Experimental Study of Wetting of the Pristine PVC Tubing}

All of the investigated extruded PVC tubing, namely pristine and plasma treated by different discharges demonstrated the partial wetting regime [35-39]. This means that the spreading parameter $S$,

$$
S=\gamma_{S A}-\left(\gamma+\gamma_{S L}\right)
$$

governing the wetting regime was negative [37-39]. In Equation (1), $\gamma=71.9 \frac{\mathrm{mJ}}{\mathrm{m}^{2}}, \gamma_{S A}$ and $\gamma_{S L}$ are interfacial tensions at water/air, PVC/air, and PVC/water interfaces, respectively. The equilibrium "as placed" equilibrium water contact angle was established at the nontreated PVC as $\theta=93 \pm 0.5^{\circ}$. This value implies the useful physico-chemical insight. Indeed, according to the Young equation, defining the equilibrium (or Young) contact angle is given by Equation (2):

$$
\cos \theta_{Y}=\frac{\gamma_{S A}-\gamma_{S L}}{\gamma}
$$

where $\gamma_{S A}, \gamma_{S L}$, and $\gamma$ are the interfacial tension at the solid/air, solid/liquid, and liquid/vapor interfaces correspondingly. It is recognized from the experimental data that the equilibrium water contact angle for PVC is close to $\theta \cong \theta_{Y} \cong \frac{\pi}{2}$. This means that $\gamma_{S A} \cong \gamma_{S L}$ takes place for PVC and this is an important conclusion because the interfacial tension at solid/liquid interfaces is not a well-known physical value. Assuming $\gamma_{S A} \cong 35-39 \frac{\mathrm{mJ}}{\mathrm{m}^{2}}[41,42]$, we conclude that for PVC $\gamma_{S L} \cong 35-39 \frac{\mathrm{mJ}}{\mathrm{m}^{2}}$ takes place. The establishment of the equilibrium contact angle enables the calculation of the specific energy of adhesion for the water/PVC pair according to the Young-Dupre formula:

$$
W \cong \gamma\left(1+\cos \theta_{Y}\right) \cong 70 \pm 1 \frac{\mathrm{mJ}}{\mathrm{m}^{2}} .
$$

The as-placed equilibrium contact angle does not exhaust the characterization of a solid surface's wettability. The advancing (maximal) $\theta_{a d v}$ and receding (minimal) $\theta_{\text {rec }}$ contact angles should be established experimentally [35-40]. Regrettably, the values of the advancing and receding contact angles depend strongly on the experimental technique used for their establishment [43,44]. Thus, we carried the measurement in parallel with the well-known "tilted plane" and "needle-syringe" methods, illustrated with Figure 2 
and 3. The maximal and minimal contact angles emerging from both of the techniques were $\theta_{a d v}=95 \pm 1^{\circ}$ and $\theta_{\text {rec }}=74 \pm 1^{\circ}$. Thus, the contact angle hysteresis established for pristine PVC as high as $\Delta \theta=\theta_{a d v}-\theta_{r e c} \cong 21^{\circ}$ was established, which is typical for the polymer surfaces [40].

Measurement of the contact angle hysteresis enabled the calculation of the solid/air surface tension (free surface energy) for PVC according to the Chibowski approach, expressed by Equation (4) and discussed in detail in [45]:

$$
\gamma_{S A}=\gamma\left(\cos \theta_{r e c}-\cos \theta_{a d v}\right) \frac{\left(1+\cos \theta_{a d v}\right)^{2}}{\left(1+\cos \theta_{r e c}\right)^{2}-\left(1+\cos \theta_{a d v}\right)^{2}} .
$$

A substitution of the aforementioned experimental values of $\theta_{a d v}$ and $\theta_{\text {rec }}$ yielded the estimation $\gamma_{S A} \cong 27.5 \pm 0.5 \frac{\mathrm{mJ}}{\mathrm{m}^{2}}$, which is lower, however, comparable with the $\gamma_{S A} \cong$ $35-39 \frac{\mathrm{mJ}}{\mathrm{m}^{2}}$ reported in [41,42]. Moreover, it practically coincides with the value of the surface energy of the extruded PVC reported recently in [46]. It is noteworthy, that the surface energy of thermoplastic polymers depends on their processing thus, the coincidence of the results, obtained for extruded PVC tubing with those reported in [46] under the same processing looks reasonable.

\subsection{Influence of the Plasma Treatment on the Wetting of PVC Tubing}

Plasma treatment of PVC tubing by all kinds of studied plasma discharges increased the hydrophilicity of the PVC catheters as shown in Figure 4. However, the Corona plasma treatment demonstrated the most pronounced influence on the wettability of catheters, as recognized from Figure 4.

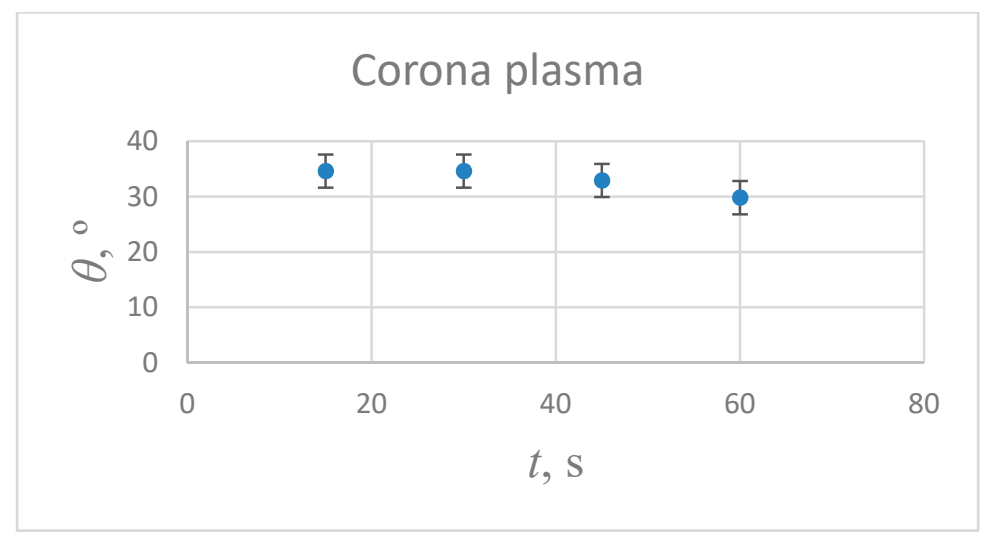

(A)

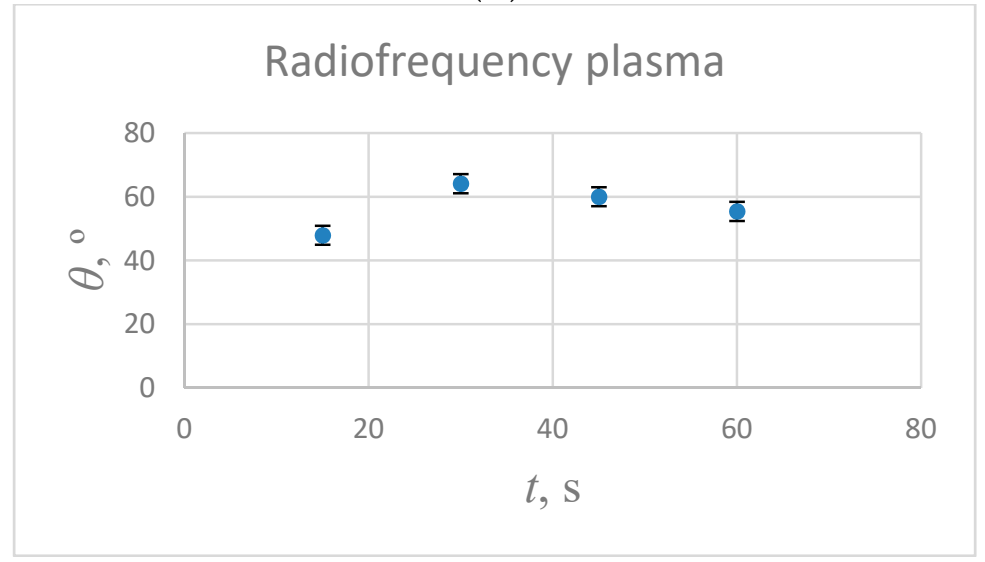

(B)

Figure 4. Cont. 


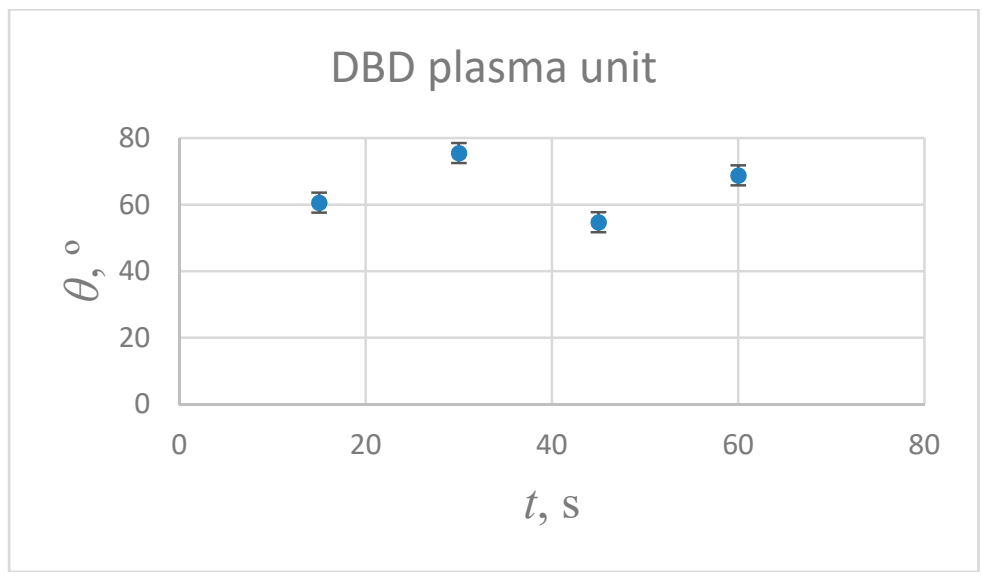

(C)

Figure 4. Impact of the dependence of the equilibrium apparent contact angle at the time of plasma treatment is presented. (A) Treatment with the Corona plasma discharge. (B) Treatment with the radiofrequency plasma device. (C) Treatment with the dielectric barrier (DBD) plasma unit.

One minute plasma treatment of the tubing by the Corona plasma discharge decreased the apparent contact angle from $\theta=93 \pm 0.5^{\circ}$ to $\theta=30 \pm 0.5^{\circ}$, whereas the apparent contact angles attained by the radiofrequency plasma discharge and DBD discharge were $\theta \cong 50-60^{\circ}$ and $\cong 60-70^{\circ}$, correspondingly. Thus, we conclude that the influence exerted by the Corona discharge on the surface properties of the PVC tubing was maximal. The increase in the energy of adhesion, following the plasma treatment as established with the Dupre formula is summarized in Table 1.

Table 1. Specific energy of adhesion established with the Dupre formula for the pristine and plasmatreated PVC tubing.

\begin{tabular}{cc}
\hline PVC Tubing & Specific Energy of Adhesion, $\boldsymbol{W}, \frac{\mathbf{m J}}{\mathbf{m}^{\mathbf{2}}}$ \\
\hline Pristine & $70 \pm 1$ \\
Corona discharge treated & $132.5 \pm 2$ \\
Radiofrequency plasma discharge treated & $111.5 \pm 5$ \\
DBD plasma discharge treated & $101.5 \pm 5$ \\
\hline
\end{tabular}

The experimentally established apparent contact angles are close to the advancing ones whereas, the receding contact angles were close to zero (namely complete wetting of the cold plasma treated PVC tubing was observed). Substitution of $\theta_{\text {adv }}=30 \pm 0.5^{\circ} ; \theta_{\text {rec }}=0$ into Equation (4) yielded an estimation of the maximal surface energy of extruded PVC tubing attainable by the cold plasma treatment as $\gamma_{S A} \cong 64.75 \pm 1 \frac{\mathrm{mJ}}{\mathrm{m}^{2}}$, which is quite expectedly essentially larger than the aforementioned free surface energy of the pristine PVC.

It is noteworthy that the impact of the radiofrequency plasma discharge and DBD discharge on the wettability of PVC comes to saturation within the first $15 \mathrm{~s}$ of the plasma treatment, whereas the influence of the Corona discharge grows slightly within the first minute of treatment, as shown in Figure 4.

\subsection{Study of the Hydrophobic Recovery Following the Plasma Treatment of the Medical Grade PVC}

The change in the surface properties of polymers induced by the cold plasma treatment is lost with time [26-30]. This effect is called the "hydrophobic recovery" [26-30]. An understanding of the kinetics and mechanisms of the hydrophobic recovery is crucial for applications of plasma-treated polymers. The pronounced hydrophobic followed the plasma treatment of plasma-treated PVC tubing as illustrated with Figures 5-7, representing 
the time evolution of the apparent contact angle measured on the plasma-treated medical grade PVC for various kinds of plasma discharges.

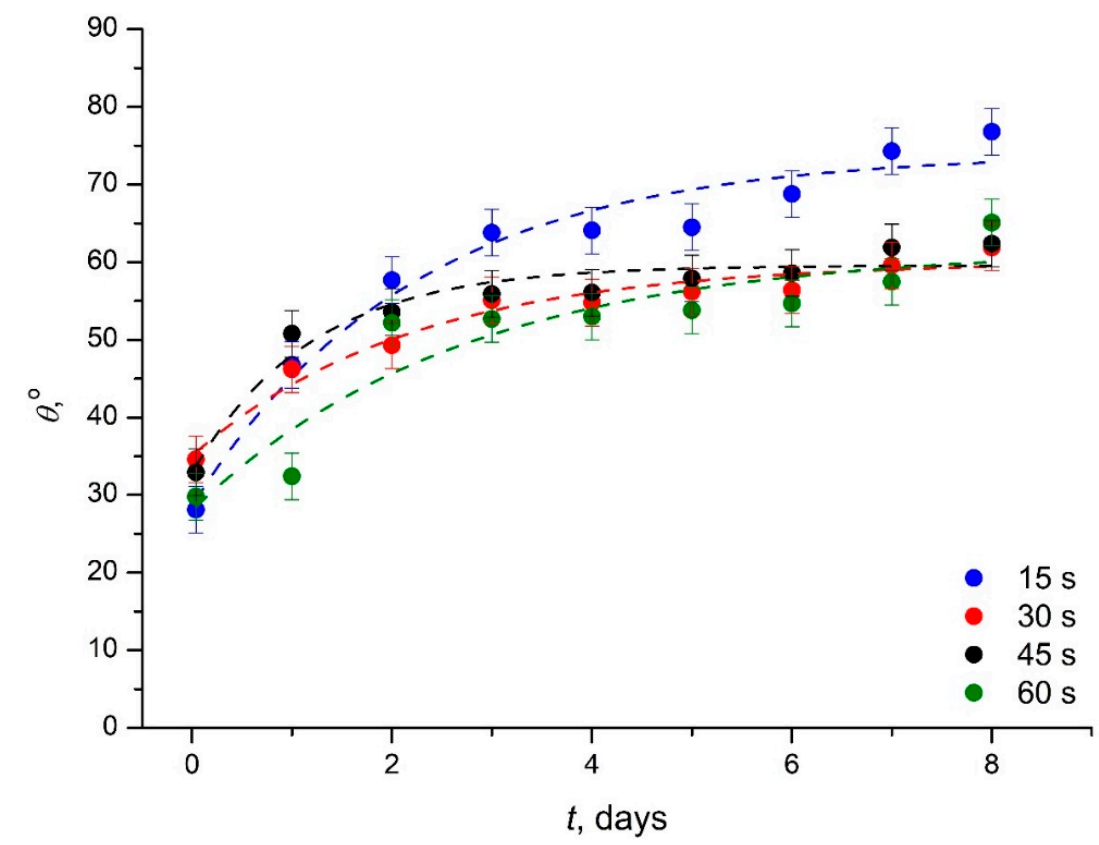

Figure 5. Kinetics of the hydrophobic recovery of PVC following Corona plasma treatment is shown. Blue circles correspond to the $15 \mathrm{~s}$ treatment; red circles correspond to the $30 \mathrm{~s}$ treatment; black circles correspond to the $45 \mathrm{~s}$ treatment; and green circles correspond to the $60 \mathrm{~s}$ treatment. The dashed line corresponds to the exponential fitting with Equation (5). The parameters of fitting are supplied in Table 2.

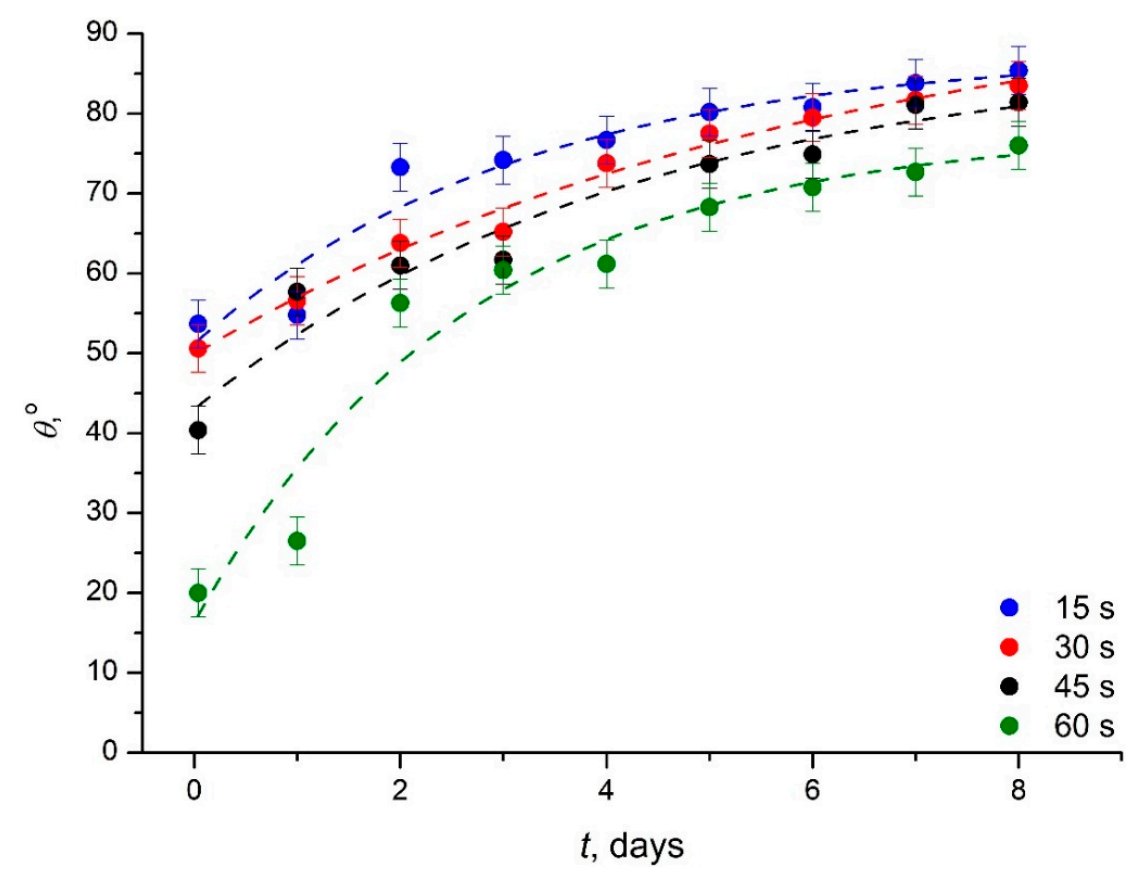

Figure 6. Kinetics of the hydrophobic recovery of PVC following the radiofrequency plasma treatment is depicted. Blue circles correspond to the $15 \mathrm{~s}$ treatment; red circles correspond to the $30 \mathrm{~s}$ treatment; black circles correspond to the $45 \mathrm{~s}$ treatment; and green circles correspond to the $60 \mathrm{~s}$ treatment. The dashed line corresponds to the exponential fitting with Equation (5). The parameters of fitting are supplied in Table 3. 
Table 2. Parameters of the exponential fitting of the kinetics of hydrophobic recovery following the Corona plasma treatment of PVC.

\begin{tabular}{cccc}
\hline Exposure Time, $\mathbf{s}$ & $\boldsymbol{\theta}_{\text {Sat }}{ }^{\circ}$ & $\widetilde{\boldsymbol{\theta}}^{\circ}$ & $\boldsymbol{\tau}$, Days \\
\hline 15 & $73 \pm 2.8$ & $45 \pm 3.7$ & $2.2 \pm 0.5$ \\
30 & $60 \pm 1.6$ & $25 \pm 2.1$ & $2.2 \pm 0.5$ \\
45 & $60 \pm 1.2$ & $27 \pm 2.6$ & $1.2 \pm 0.3$ \\
60 & $62 \pm 5.3$ & $34 \pm 5.7$ & $2.7 \pm 1.3$ \\
\hline
\end{tabular}

Table 3. Parameters of the exponential fitting of the kinetics of hydrophobic recovery following the radiofrequency plasma treatment of PVC.

\begin{tabular}{cccc}
\hline Exposure Time, $\mathbf{s}$ & $\boldsymbol{\theta}_{\text {Sat }}{ }^{\circ}$ & $\widetilde{\boldsymbol{\theta}}^{\circ}{ }^{\circ}$ & $\boldsymbol{\tau}$, Days \\
\hline 15 & $87 \pm 5.0$ & $36 \pm 4.8$ & $3.1 \pm 1.2$ \\
30 & $97 \pm 6.8$ & $47 \pm 6.2$ & $6 \pm 1.6$ \\
45 & $88 \pm 8.7$ & $45 \pm 7.7$ & $4.3 \pm 1.9$ \\
60 & $78 \pm 5.7$ & $62 \pm 6.2$ & $2.7 \pm 0.8$ \\
\hline
\end{tabular}

Table 4. Parameters of the exponential fitting of the kinetics of hydrophobic recovery following the DBD treatment of PVC.

\begin{tabular}{cccc}
\hline Exposure Time, $\mathbf{s}$ & $\boldsymbol{\theta}_{\text {Sat }}$ & $\widetilde{\boldsymbol{\theta}}$ & $\boldsymbol{T}$, Days \\
\hline 15 & $86 \pm 1.2$ & $27 \pm 2.7$ & $0.7 \pm 0.2$ \\
30 & $88 \pm 0.2$ & $13 \pm 0.3$ & $1.3 \pm 0.1$ \\
45 & $83 \pm 2.2$ & $31 \pm 5.6$ & $0.5 \pm 0.3$ \\
60 & $85 \pm 2.5$ & $17 \pm 3.4$ & $1.5 \pm 0.1$ \\
\hline
\end{tabular}

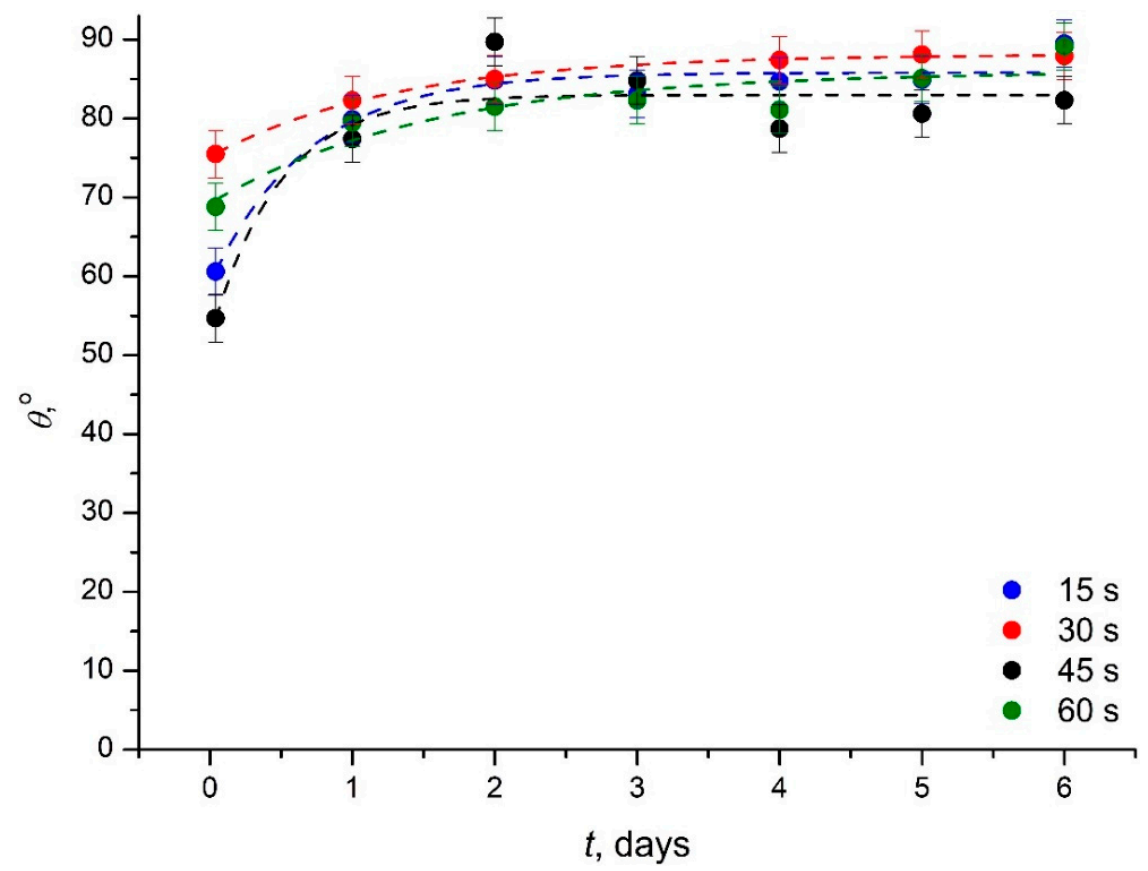

Figure 7. Kinetics of the hydrophobic recovery of PVC following the DBD plasma treatment is depicted. Blue circles correspond to the $15 \mathrm{~s}$ treatment; red circles correspond to the $30 \mathrm{~s}$ treatment; black circles correspond to $45 \mathrm{~s}$ treatment; and green circles correspond to the $60 \mathrm{~s}$ treatment. The dashed line corresponds to the exponential fitting with Equation (5). The parameters of fitting are supplied in Table 4. 
The kinetics of hydrophobic recovery is satisfactorily described by the exponential fitting, suggested in [28]:

$$
\theta(t)=\widetilde{\theta}\left(1-e^{-\frac{t}{\tau}}\right)+\theta_{0}=\theta_{s a t}-\widetilde{\theta} e^{-\frac{t}{\tau}}
$$

where $\theta_{0}$ is the initial apparent contact angle, taken immediately after the plasma treatment of PVC catheters, $\tau$ is the characteristic time of restoring the contact angle, $\widetilde{\theta}$ is the fitting parameter, and $\theta_{\text {sat }}=\widetilde{\theta}+\theta_{0}$ is the saturation apparent contact angle. The numerical values of the parameters of the exponential fitting for the investigated plasma discharges are supplied in Tables $2-4$.

A number of common features are recognized for the hydrophobic recovery of PVC tubing exposed to various plasma discharges: (i) The hydrophobic recovery following the plasma treatment is well described by the exponential fitting for all kinds of the studied discharges and (ii) the saturation contact angle $\theta_{\text {sat }}$ is slightly lower than the initial apparent contact angle, in other words, the hydrophobic recovery following the plasma treatment of PVC is never complete.

On the other hand, the characteristic time of the hydrophobic recovery is largest for the radiofrequency plasma discharge, namely $\tau \cong 3.0-6.0$ days; it is intermediate for the Corona discharge, i.e., $\tau \cong 1.0-2.5$ days; and it is small for the DBD discharge, namely: $\tau \cong 0.5-1.5$ days. Thus, the hydrophobic recovery following the cold plasma treatment is the slowest for the radiofrequency plasma discharge and it is rapid for the DBD plasma treatment. At this stage of our investigation we are far from the microscopic interpretation of these experimental findings, which are of a primary importance for the applications of the plasma processing of PVC, however some conclusions may be made from the energy-dispersive X-ray spectroscopy (abbreviated further EDS spectroscopy) of the plasma-treated PVC catheters, discussed in the following section.

\subsection{EDS Analysis of the Chemical Composition of the Plasma Treated PVC Catheters}

The EDS study of the chemical composition of the pristine and plasma-treated PVC catheters indicated the growth of the concentration of oxygen in the near surface layers of plasma-treated PVC catheters, as demonstrated in Figure 8. The presence of oxygen in the pristine PVC is reasonably explained by its oxidation under high-temperature extrusion processing [47].

The growth of the concentration of oxygen from 7 to 9 atomic percent was registered, as shown in Figure 8. It should be emphasized that this tendency was kept for all kinds of investigated plasma discharges. The concentration of oxygen in the surface layer of catheters grew with the time of treatment, as shown in Figure 8. These results coincide with the finding reported by the other research groups [48]. It was demonstrated that when PVC is treated with argon plasma, unsaturated bonds are created at the surface [49]. It was also shown that the pendant groups of PVC are removed by the argon plasma treatment [49]. This resulted in the formation of unsaturated bonds and cross-links in the modified layer and the outermost top layer of the polymer became oxidized after exposure to air due to a reaction between long-living radicals and oxygen [49]. It is reasonable to suggest that the similar mechanism also takes place under the cold air plasma treatment, thus, giving rise to the change in the wettability of PVC, discussed in detail in the previous sections. 


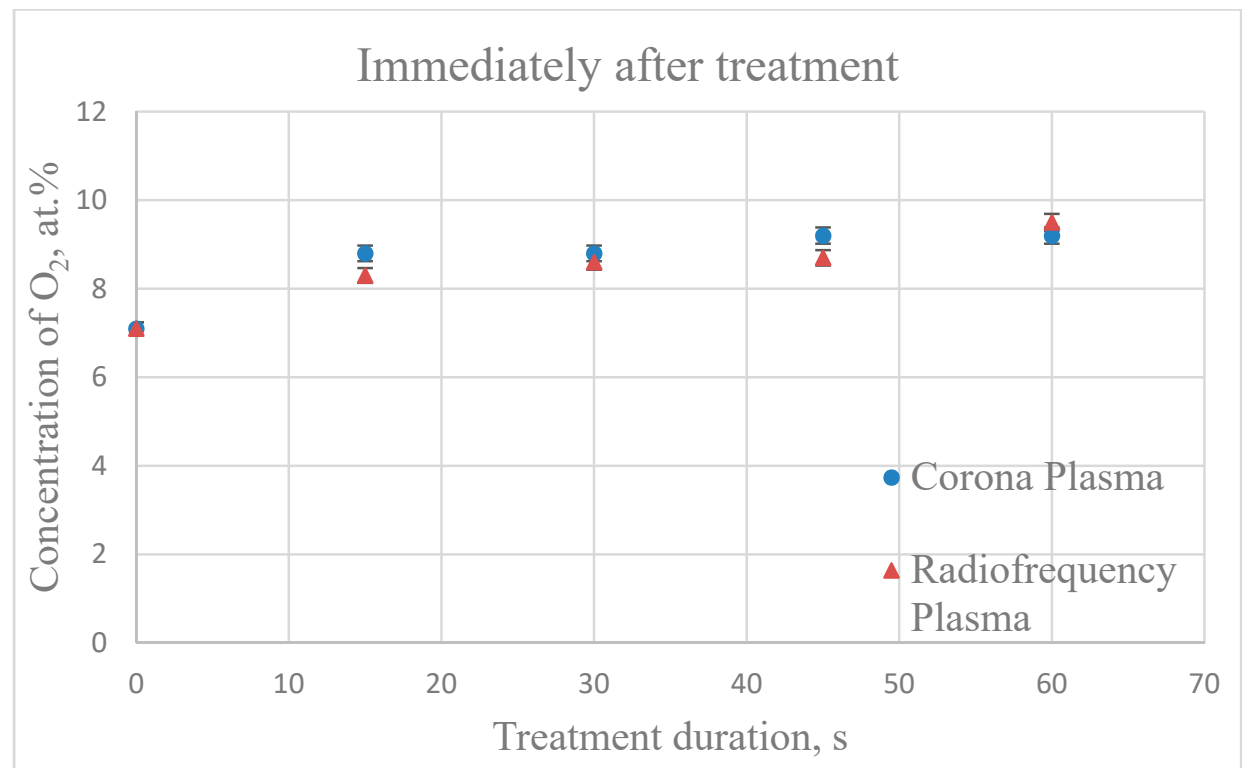

Figure 8. The surface concentration of oxygen (atomic percent) in the surface layer of catheters vs. the duration of plasma treatment as established immediately after the treatment with SEM/EDS spectroscopy is depicted.

It was instructive to study the surface composition of the plasma-treated PVC catheters after one week of ageing under ambient conditions. It is recognized from the data presented in Figure 9 and its comparison to the data shown in Figure 8, that the concentration of oxygen in the near-surface layers of the plasma-treated catheters decreased very slightly. This observation is quite expectable as oxidation of PVC emerging from the plasma treatment is an irreversible process $[48,49]$. Consider that the time of ageing was markedly larger that the characteristic time of the hydrophobic recovery, supplied in Table 1. Thus, we conclude, that the process of the hydrophobic recovery hardly correlated with the oxidation of the polymer arising from the cold plasma treatment.

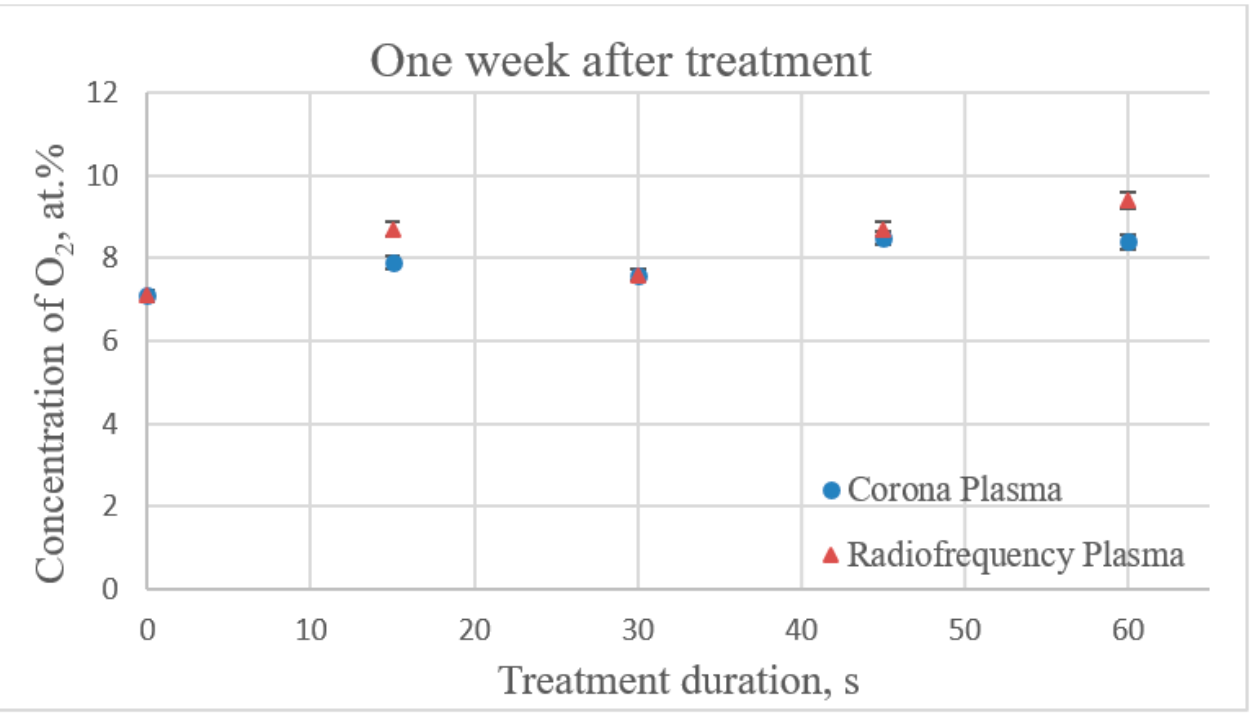

Figure 9. The surface concentration of oxygen (atomic percent) in the surface layer of catheters vs. the time of plasma treatment as established with SEM/EDS spectroscopy one week after the treatment is presented.

Hydrophobic recovery is a complex relaxation process including restoring of mechanical, electrical, and surface properties of plasma-treated polymers [50]. The relatively 
"long" times inherent to the kinetics of the hydrophobic recovery of the plasma-treated PVC ( $\tau \cong 1.0$ day) hint to the assumption that the effect is constituted to a large extent by the bulk mobility of polymer chains [50]. The surface mobility of polymer chains is described by the markedly shorter characteristic times [50].

\section{Conclusions}

Cold plasma treatment is broadly used for modification of chemical composition and wettability of organic and nonorganic compounds [1-9,51]. The influence of the plasma treatment on the surface chemical composition and properties of the polyvinylchloride catheters was explored with three different plasma sources. It can be concluded that the Corona, low pressure radiofrequency, and DBD plasma discharges increased essentially the hydrophilicity of PVC tubing. The Corona plasma treatment demonstrated the most pronounced influence on the eventual wettability of catheters. One-minute plasma treatment of the tubing by the Corona plasma discharge decreased the apparent contact angle from $\theta=93 \pm 0.5^{\circ}$ to $\theta=30 \pm 0.5^{\circ}$. The energy of water adhesion of pristine and plasma-treated PVC estimated with the Dupre formula is reported. Cold plasma treatment increased markedly the specific free surface energy of PVC, calculated with the Chibowski equation [45]. Kinetics of the hydrophobic recovery following the plasma treatment of PVC was explored [26-30]. The hydrophobic recovery following the plasma treatment of PVC is never complete, in other words the saturation contact angle $\theta_{\text {sat }}$ is slightly lower than the initial apparent contact angle inherent for the pristine PVC. It is noteworthy that the kinetics of hydrophobic recovery following the plasma treatment is well-described by the exponential fitting suggested in [28] for all kinds of studied discharges. We established that the hydrophobic recovery following the cold plasma treatment was the slowest for the radiofrequency plasma discharge, and was most rapid for the DBD plasma treatment of PVC. The characteristic times inherent to the kinetics of the hydrophobic recovery of the plasma-treated PVC ( $\tau \cong 1.0$ day) supported the hypothesis that the effect was influenced by the bulk mobility of polymer chains [50]. Energy-dispersive X-ray spectroscopy of the chemical composition of the near-surface layers of the catheters indicated their oxidation, emerging from the cold air plasma treatment. This oxidation was irreversible and changed very slightly under one week ageing under ambient conditions. Thus, we conclude that the phenomenon of the hydrophobic recovery hardly correlated with the oxidation of the polymer (PVC) surface.

Author Contributions: Conceptualization, E.B. and S.N.-V.; methodology, I.L. and M.F.; validation, M.F. and Y.B.; formal analysis, I.L. and M.F.; investigation, I.L. and Y.B.; resources, Y.B.; data curation, I.L. and Y.B.; writing-original draft preparation, E.B.; writing-review and editing, E.B. and S.N.-V.; supervision, E.B. and S.N.-V.; project administration, S.N.-V.; funding acquisition, S.N.-V. All authors have read and agreed to the published version of the manuscript.

Funding: This research was supported by the Ministry of Science \& Technology, Israel (Grant No. 3-16288) supporting EB and SNV.

Institutional Review Board Statement: Not applicable.

Informed Consent Statement: Not applicable.

Data Availability Statement: The data presented in this study are available on request from the corresponding author.

Acknowledgments: This work was supported by a grant from the Israel Ministry of Science and Technology, Grant no Merkava 3-16288 supporting EB and SNV. The authors are indebted to N. Litvak for her kind help with the energy-dispersive X-ray spectroscopy analysis of the samples. The authors are indebted to the anonymous reviewers for extremely fruitful suggestions.

Conflicts of Interest: The authors declare no competing interest. 


\section{References}

1. Yasuda, H.; Gazicki, M. Biomedical applications of plasma polymerization and plasma treatment of polymer surfaces. Biomaterials 1982, 3, 68-77. [CrossRef]

2. Oehr, C. Plasma surface modification of polymers for biomedical use. Nucl. Instrum. Meth. Phys. Res. B 2003, 208, 40-47. [CrossRef]

3. Gomathi, N.; Sureshkumar, A.; Neogi, S. RF plasma-treated polymers for biomedical applications, Current Science. Curr. Sci. 2008, 94, 1478-1486.

4. Cheruthazhekatt, S.; Černák, M.; Slavíček, P.; Havel, J. Gas plasmas and plasma modified materials in medicine. J. Appl. Biomed. 2010, 8, 55-66. [CrossRef]

5. Joshy, K.S.; Snigdha, S.; Thomas, S. Plasma Modified Polymeric Materials for Scaffolding of Bone Tissue Engineering. In NonThermal Plasma Technology for Polymeric Materials; Thomas, S., Mozetič, M., Cvelbar, U., Špatenka, P., Praveen, K.M., Eds.; Elsevier: Amsterdam, The Netherlands, 2019; pp. 439-458.

6. Grace, J.M.; Gerenser, L.J. Plasma treatment of polymers. J. Dispers. Sci. Technol. 2003, 24, 305-341. [CrossRef]

7. Strobel, M.; Lyons, C.S.; Mittal, K.L. (Eds.) Plasma Surface Modification of Polymers: Relevance to Adhesion; VSP: Zeist, The Netherlands, 1994.

8. Thomas, M.; Mittal, K.L. (Eds.) Atmospheric Pressure Plasma Treatment of Polymers, Plasma Surface Modification of Polymers: Relevance to Adhesion; Scrivener Publishing: Beverly, MA, USA, 2013.

9. Yasuda, H.K. (Ed.) Plasma Polymerization and Plasma Treatment; J. Wiley \& Sons: New York, NY, USA, 1984.

10. France, R.M.; Short, R.D. Plasma treatment of polymers: The Effects of energy transfer from an Argon plasma on the surface chemistry of polystyrene, and polypropylene. A High-Energy resolution X-ray photoelectron spectroscopy study. Langmuir 1998, 14, 4827-4835. [CrossRef]

11. France, R.M.; Short, R.D. Plasma treatment of polymers: Effects of energy transfer from an argon plasma on the surface chemistry of poly(styrene), low density poly(ethylene), poly(propylene) and poly(ethylene terephthalate). J. Chem. Soc. Faraday Trans. 1997, 93, 3173-3178. [CrossRef]

12. Wild, S.; Kesmodel, L.L. High resolution electron energy loss spectroscopy investigation of plasma-modified polystyrene surfaces. J. Vac. Sci. Technol. A 2001, 19, 856-860. [CrossRef]

13. Gaiser, S.; Schütz, U.; Hegemann, D. Top-down approach to attach liquid polyethylene glycol to solid surfaces by plasma interaction. Plasma Process. Polym. 2020, 7, 1900211. [CrossRef]

14. Bormashenko, E.; Whyman, G.; Multanen, V.; Shulzinger, E.; Chaniel, G. Physical mechanisms of interaction of cold plasma with polymer surfaces. J. Colloid Interface Sci. 2015, 448, 175-179. [CrossRef]

15. Shapira, Y.; Chaniel, G.; Bormashenko, E. Surface charging by the cold plasma discharge of lentil and pepper seeds in comparison with polymers. Colloids Surf. B. 2018, 172, 541-544. [CrossRef] [PubMed]

16. Lehocky, M.; Drnovska, H.; Lapcikova, B.; Barros-Timmons, A.M.; Trindade, T.; Zembala, M.; Lapcik, L., Jr. Plasma surface modification of polyethylene. Colloids Surf. A 2003, 222, 125-131. [CrossRef]

17. Preedy, E.C.; Brousseau, E.; Evans, S.L.; Perni, S.; Prokopovich, P. Adhesive forces and surface properties of cold gas plasma treated UHMWPE. Colloids Surf. A. 2014, 460, 83-89. [CrossRef] [PubMed]

18. Hegemann, D.; Brunner, H.; Oehr, C. Plasma treatment of polymers for surface and adhesion improvement. Nucl. Instrum. Methods Phys. Res. B 2003, 208, 281-286. [CrossRef]

19. Correia, D.M.; Nunes-Pereira, J.; Alikind, D.; Kholkin, A.L.; Carabineiro, S.A.C.; Rebouta, L.; Rodrigues, M.S.; Vaz, F.; Costa, C.M.; Lanceros-Méndez, S. Surface wettability modification of poly(vinylidene fluoride) and copolymer films and membranes by plasma treatment. Polymer 2019, 169, 138-147. [CrossRef]

20. Balu, B.; Breedveld, V.; Hess, D.W. Fabrication of "roll-off" and "sticky" superhydrophobic cellulose surfaces via plasma processing. Langmuir 2008, 24, 4785-4790. [CrossRef]

21. Bormashenko, E.; Eldar, B.; Chaniel, G.; Multanen, V.; Whyman, G. Influence of cold radiofrequency air and nitrogen plasmas treatment on wetting of polypropylene by the liquid epoxy resin. Colloids Surf. A 2016, 506, 445-449. [CrossRef]

22. Kaminska, A.; Kaczmarek, H.; Kowalonek, J. The influence of side groups and polarity of polymers on the kind and effectiveness of their surface modification by air plasma action. Eur. Polym. J. 2002, 38, 1915-1919. [CrossRef]

23. Tan, S.H.; Nguyen, N.-T.; Chua, Y.C.; Kang, T.G. Oxygen plasma treatment for reducing hydrophobicity of a sealed polydimethylsiloxane microchannel. Biomicrofluidics 2010, 4, 032204. [CrossRef]

24. Foerch, R.; McIntyre, N.S.; Hunter, D.H. Oxidation of polyethylene surfaces by remote plasma discharge: A comparison study with alternative oxidation methods. J. Polym. Sci. A 1990, 28, 193-204. [CrossRef]

25. Ladner, Y.; D'Orlye, F.; Perreard, C.; Da Silva, B.; Guyon, C.; Tatoulian, M.; Griveau, S.; Bedioui, F.; Varenne, A. Surface functionalization by plasma treatment and click chemistry of a new family of fluorinated polymeric materials for microfluidic chips. Plasma Process. Polym. 2014, 11, 518-523. [CrossRef]

26. Occhiello, E.; Morra, M.; Garbassi, F.; Johnson, D.; Humphrey, P. SSIMS studies of hydrophobic recovery: Oxygen plasma treated PS. Appl. Surf. Sci. 1991, 47, 235-242. [CrossRef]

27. Pascual, M.; Kerdraon, M.; Rezard, Q.; Jullien, M.-C.; Champougny, L. Wettability patterning in microfluidic devices using thermally-enhanced hydrophobic recovery of PDMS. Soft Matter 2019, 15, 9253-9260. [CrossRef] [PubMed] 
28. Bormashenko, E.; Chaniel, G.; Grynyov, R. Towards understanding hydrophobic recovery of plasma treated polymers: Storing in high polarity liquids suppresses hydrophobic recovery. Appl. Surf. Sci. 2013, 273, 549-553. [CrossRef]

29. Hegemann, D.; Lorusso, E.; Butron-Garcia, M.-I.; Blanchard, N.E.; Rupper, P.; Favia, P.; Heuberger, M.; Vandenbossche, M. Suppression of Hydrophobic Recovery by Plasma Polymer Films with Vertical Chemical Gradients. Langmuir 2016, 32, 651-654. [CrossRef]

30. Mortazavi, M.; Nosonovsky, M. A model for diffusion-driven hydrophobic recovery in plasma treated polymers. Appl. Surf. Sci. 2012, 258, 6876-6883. [CrossRef]

31. Ru, L.; Jie-rong, C. Studies on wettability of medical poly(vinyl chloride) by remote argon plasma. Appl. Surf. Sci. 2006, 252, 5076-5082. [CrossRef]

32. Zhang, W.; Chu, P.K.; Jia, J.; Zhang, Y.; Liu, X.; Fu, R.K.Y.; Ha, P.C.T.; Yan, Q. Plasma surface modification of poly vinyl chloride for improvement of antibacterial properties. Biomaterials 2006, 27, 44-51. [CrossRef]

33. Ghoranneviss, M.; Shahidi, S.; Wiener, J. Surface Modification of Poly Vinyl Chloride (PVC) Using Low Pressure Argon and Oxygen Plasma. Plasma Sci. Technol. 2010, 12, 204-207. [CrossRef]

34. Fazekas, P.; Keszler, A.M.; Bódis, E.; Drotár, E.; Klébert, S.; Károly, Z.; Szépvölgy, J. Optical emission spectra analysis of thermal plasma treatment of poly(vinyl chloride). Open Chem. 2015, 13, 549-556. [CrossRef]

35. Tadmor, R. Line Energy and the Relation between Advancing, Receding, and Young Contact Angles. Langmuir 2004, 20, 7659-7664. [CrossRef] [PubMed]

36. Tadmor, R.; Yadav, P.S. As-placed contact angles for sessile drops. J. Colloid Interface Sci. 2008, 317, 241-246. [CrossRef] [PubMed]

37. De Gennes, P.G.; Brochard-Wyart, F.; Quéré, D. Capillarity and Wetting Phenomena; Springer: Berlin, Germany, 2003.

38. Erbil, H.Y. Surface Chemistry of Solid and Liquid Interfaces; Blackwell Publishing: Oxford, UK, 2006.

39. Bormashenko, E. Wetting of Real Surfaces; de Gruyter: Berlin, Germany, 2013.

40. Extrand, C.W.; Kumagai, Y. An Experimental Study of Contact Angle Hysteresis. J. Colloid Interface Sci. 1997, 191, 378-383. [CrossRef]

41. Van Krevelen, D.W. Properties of Polymers, Their Correlation with Chemical Structure. In Their Numerical Estimation and Prediction from Additive Group Contributions, 4th ed.; Elsevier: Amsterdam, The Netherlands, 2009.

42. Jierong, C.; Jing-Lian, Y.; Yun-Ze, Z. Surface modification of medical PVC by remote oxygen plasma. Compos. Interfaces 2004, 11, 123-130. [CrossRef]

43. Chibowski, E.; Perea-Carpio, R. Problems of contact angle and solid surface free energy determination. Adv. Colloid Interface Sci. 2002, 98, 245-264. [CrossRef]

44. Bormashenko, E.; Bormashenko, Y.; Whyman, G.; Pogreb, R.; Musin, A.; Jager, R.; Barkay, Z. Contact angle hysteresis on polymer substrates established with various experimental techniques, its interpretation, and quantitative characterization. Langmuir 2008, 24, 4020-4025. [CrossRef]

45. Chibowski, E. Surface free energy of a solid from contact angle hysteresis. Adv. Colloid Interface Sci. 2003, 103, 149-172. [CrossRef]

46. Garbacz, T. Surface free energy of extruded polymer compositions. Physicochem. Probl. Miner. Process. 2019, 55, $1509-1516$.

47. Nagy, T.T.; Kelen, T.; Turcsányi, B.; Tüdös, F. Initiated oxidation of polyenes formed in the thermal degradation of PVC. J. Polym. Sci. 1977, 15, 853-864. [CrossRef]

48. Kaczmarek, H.; Kowalonek, J.; Szalla, A.; Sionkowska, A. Surface modification of thin polymeric films by air-plasma or UV-irradiation. Surf. Sci. 2002, 507-510, 883-888. [CrossRef]

49. Groenewoud, L.M.H.; Terlingen, J.G.A.; Engbers, G.H.M.; Feijen, J. Removal of Pendant Groups of Vinyl Polymers by Argon Plasma Treatment. Langmuir 1999, 15, 5396-5402. [CrossRef]

50. Pogreb, R.; Loew, R.; Bormashenko, E.; Whyman, G.; Multanen, V.; Shulzinger, E.; Abramovich, A.; Rozban, D.; Shulzinger, A.; Zussman, E.; et al. Relaxation Spectra of Polymers and Phenomena of Electrical and Hydrophobic Recovery: Interplay between Bulk and Surface Properties of Polymers. J. Polym. Sci. B 2017, 55, 198-205. [CrossRef]

51. Yu, H.; Gong, L.; Qu, Z.; Hao, P.; Liu, J.; Fu, L. Wettability enhancement of hydrophobic artificial sandstones by using the pulsed microwave plasma jet. Colloid Interface Sci. Commun. 2020, 36, 100266. [CrossRef] 